\title{
The Three-Dimensional Architecture of the Cribriform Pattern in Adenoid Cystic Carcinoma of Salivary Gland Origin
}

Ken Onodera and Kiyoshi Ooya

Division of Oral Pathology, Department of Oral Medicineand Bioregulation, Tohoku University GraduateSchool of Dentistry, Sendai, J apan Onodera $K$ and Ooya $K$. The three-dimensional architecture of the cribriform pattern in ade-
noid cystic carcinoma of salivary gland origin. Oral Med Pathol 2000; 5: 77-81, ISSN 1342-0984.

The three-dimensional (3-D) architecture of the cribriform pattern in adenoid cystic carcinoma of salivary gland origin was studied by histological and immunohistochemical findings. Tumor cell nests had numerous spaces composed of both laminin negative true cystic spaces and laminin positive pseudocystic spaces with occasional interconnection to the outer stromal tissue by light microscope. By the 3-D architecture, in tumor cell nests with predominant pseudocyst, the true cystic spaces showed the isolated spherical shape, whereas the pseudocystic spaces showed the spherical and/or ovoid shape interconnected with other pseudocystic spaces. The mean number of argyrophilic nucleolar organizer regions (AgNORs) per nucleus of the tumor cells forming the pseudocyst was significantly higher than that forming the true cyst. This study supports that these 3-D features were useful for a better understanding of biological characteristics of epithelial tumor cell nests of adenoid cystic carcinoma.

Key words: Adenoid cystic carcinoma, Cribriform, Three-dimensional architecture, AgNORs

Correspondence: Ken Onodera, Division of Oral Pathology, Department of Oral Medicine and Bioregulation, Tohoku University Graduate School of Dentistry,4-1 Seiryo-machi, Aoba, Sendai, Miyagi, 980-8575, J apan

\section{Introduction}

Adenoid cystic carcinoma (ACC) is the wellrecognized malignant tumor of exocrine glands, first de scribed by Billroth (1) in 1859 as the name "Zylindrome" (cylindroma) because the histological appearance of this tumor was similar to thyroid follicles composed of cylinders of hyalin and mucin surrounded by tubes of the epithelial elements. Many different names have been proposed such as "cribriform adenocarcinoma" (2), "basalioma" (3), and "adenocarcinoma of mixed tumor type" (4), although the term "cylindroma" is still in common use. More recently, however, the WHO subcommittee on classification of tumors recommended adoption of the term "adenoid cystic carcinoma", which was first used by Reid (5). One of the most characteristic and classical features of ACC is the cribriform pattern showing numerous cystic spaces surrounded by anastomosing cord of epithelial tumor cells, and that is considered to be an important factor responsible for the biological behavior of this tumor. Although this peculiar cystic feature has been described as "sieve", "cylinder", and "Swiss cheese" by light microscope, no stereographic architecture has been elucidated.

In order to clarify the morphological characteristics of the cribriform pattern in ACC, a recent, advanced stereographic computer-technology was applied for inves- tigating the three-dimensional (3-D) structural attributes of ACC. Furthermore, the proliferative status of tumor cells constituting cystic spaces was evaluated to investigate the relation to cyst formation.

\section{Materials and Methods}

A total five cases of the cribriform pattern in ACC of salivary gland origin were retrieved from surgical pathology files of Tohoku University Graduate School of Dentistry (Sendai, J apan) for the present study. They were three women and two men of ages ranging from 56 to 76 years, with ACC originating in four minor salivary glands and one sublingual gland. Biopsy specimens had been fixed in $10 \%$ formalin solution, dehydrated in graded ethanol solutions and xylene, and embedded in paraffin. Over 200 serial sections of each specimen were made in a thickness of about $3 \mu \mathrm{m}$ with a microtome and mounted on glass slides. Hematoxylin and eosin (HE) staining, and argyrophilic nucleolar organizer region (AgNOR) staining (6) were performed. For AgNOR staining, paraffin sections were dewaxed in xylene and then hydrated through a graded series of ethanol to water. The AgNOR staining solution was a $2 \%$ solution of gelatin in $1 \%$ formic acid. One volume of this solution was combined with two volumes of $50 \%$ silver nitrate solution. The prepared solu- 
tion was poured over the tissue sections and allowed to stand 30 minutes in a darkened room. The sections were then washed with deionized water, dehydrated to xylene, and mounted.

\section{Immunohistochemical staining}

Following deparaffinisation of sections, immunohistochemical staining was performed using antibodies directed against laminin (dilution 1:100, DAKO, Kyoto, J apan), muscle-specific actin (dilution 1:100, clone HHF 35, DAKO, Kyoto, J apan) and cytokeratin (dilution 1:200, clone AE 1/AE 3, Becton-Dickinson, San J ose, USA). Bound antibodies were visualized using the supersensitive streptavidin-biotin detection system (Biogenex, San Ramon, USA). The color was developed with diaminobenzidine and supplemented with hydrogen peroxide. The sections were finally counterstained with Mayer's hematoxylin.

Thre-dimensional reconstruction

Representative sections were selected for the 3-D

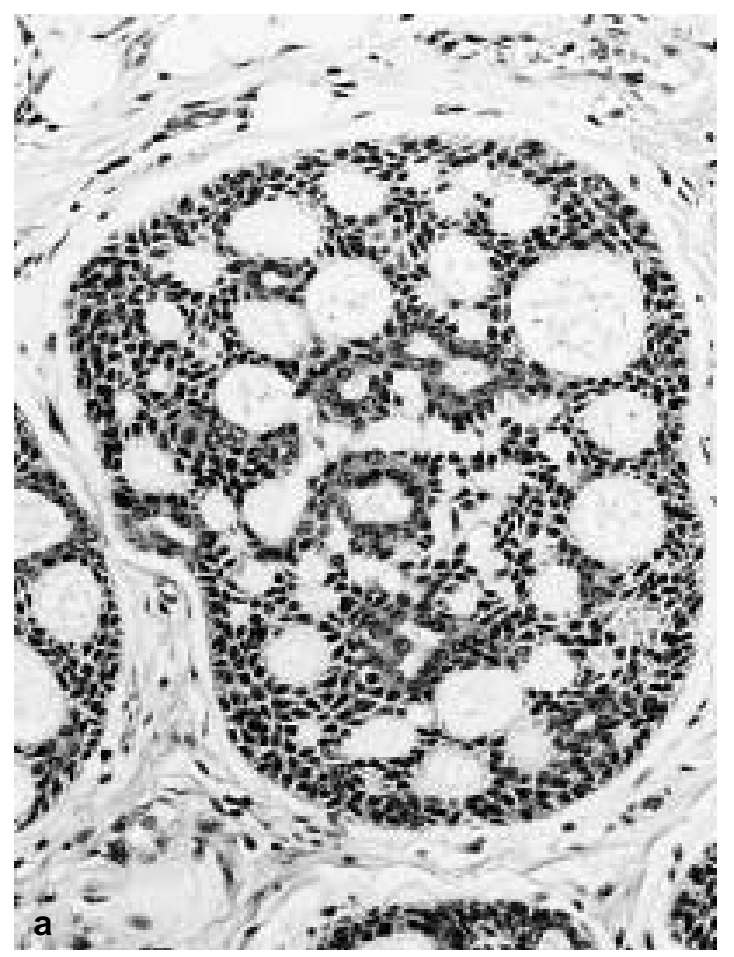

Fig. 1: (a) Cribriform pattern in ACC with numerous cystic spaces in the tumor nest. $(\mathrm{HE}, \times 160)(\mathrm{b}, \mathrm{c})$ Two types of the cystic structure. (HE, ×540) (b) Surrounding one or two layers of cuboidal and polygonal tumor cells. (c) Surrounding rather small polygonal and spindle-shape tumor cells. reconstruction using a computer graphics analysis system ('TRI-P' Ratoc System Engineering Co., Ltd, Tokyo) after $\mathrm{HE}$, AgNOR and immunohistochemical staining. A $35 \mathrm{~mm}$ black and white photographic serial film strip was prepared. The serial photographic images of the outlines of tumor cell nests and cystic spaces of ACC then traced manually on the computer screen were synthesized into computer-generated 3-D images suitable for examination from different perspectives.

\section{Results}

HE findings

The cribriform pattern in ACC was characterized by numerous cystic structures (Fig.1-a). An abundance of mucinous substances were packed within the cystic spaces surrounded by tumor cells. The outer stroma surrounding the tumor cells nests was composed of dense fibrous connective tissue containing fibroblasts, collagen fibers and blood vessels. There were two types of cystic structures; one was surrounded by one or two layers of cuboidal and polygonal tumor cells (Fig.1-b), and the
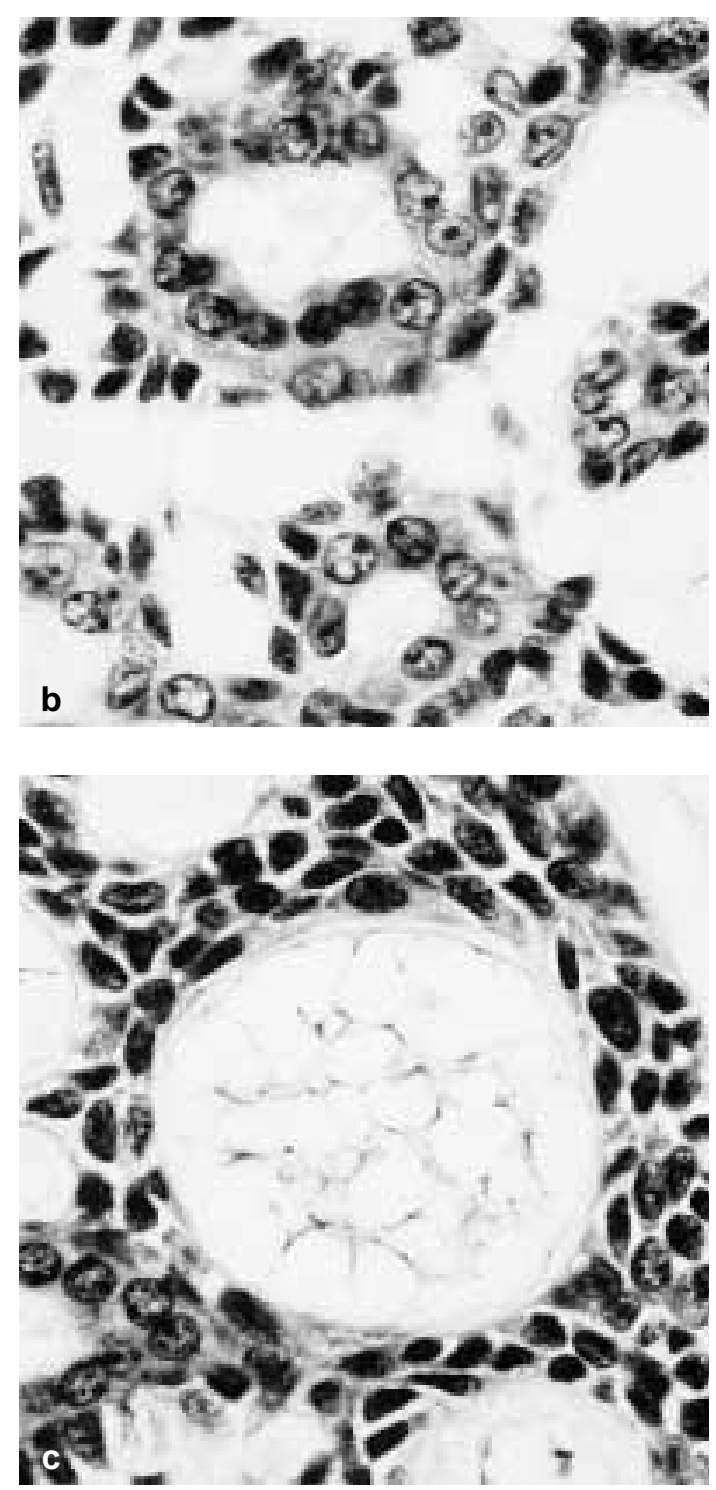
other was surrounded by rather small polygonal and spindle-shape tumor cells (Fig.1-c). In some areas, the tumor cell nests were broken up to form thin strands, and the cystic spaces were connected more frequently to one another and the interstitial connective tissue surrounding tumor nests.

\section{Immunohistochemical findings}

Laminin staining was characterized by its presence in the basement membrane of the tumor nests and cystic spaces (Fig.2). The inner luminal surface surrounded by rather small polygonal and spindle-shape tumor cells strongly reacted for laminin, whereas that surrounded by cuboidal and polygonal tumor cells of one or two layers was negative (Fig.2). Furthermore, cuboidal and polygonal tumor cells of one or two layers were generally positive for cytokeratin and negative for muscle-specific actin, whereas rather small polygonal and spindle-shape tumor cells were usually negative for cytokeratin and positive for muscle-specific actin. These immunohistochemical results, as emphasized in prior literature (7-9), indicated that one or two layers of cuboidal and polygonal tumor cells were ductal epithelium-like cells forming the true cyst, whereas rather small polygonal and spindle-shape tumor cells were myoepithelium-like cells forming the pseudocyst.

Threedimensional findings

In the tumor cell nest with predominant pseudocyst (Fig.3, inset), the true cystic spaces showed the isolated spherical shape in the tumor nest, as visualized in Fig.3a. The pseudocystic spaces showed the spherical and/or ovoid shape of varying sizes, as visualized in Fig.3-b. Many of the pseudocystic spaces were interconnected with each other.

\section{AgNORs findings}

AgNORs were observed within nuclei as black dots with a lighter background (Fig.4-a). Tumor cells containing 1 or 2 AgNOR dots were predominant in the true cyst (Fig.4-a, left), whereas tumor cells containing 2 or more AgNOR dots were widespread in the pseudocyst (Fig.4-a, right). The mean number of AgNORs per nucleus (AgNOR number) of tumor cells constituting true cysts like Fig.3-a and various sized pseudocysts like Fig.3-b was 1.6 $\pm 0.7(n=194)$ and $3.1 \pm 1.2(n=440)$, respectively (Fig.4-b). It was significantly higher in the tumor cells constituting pseudocysts than in those that constituting true cysts $(p \triangleleft 0.001)$.
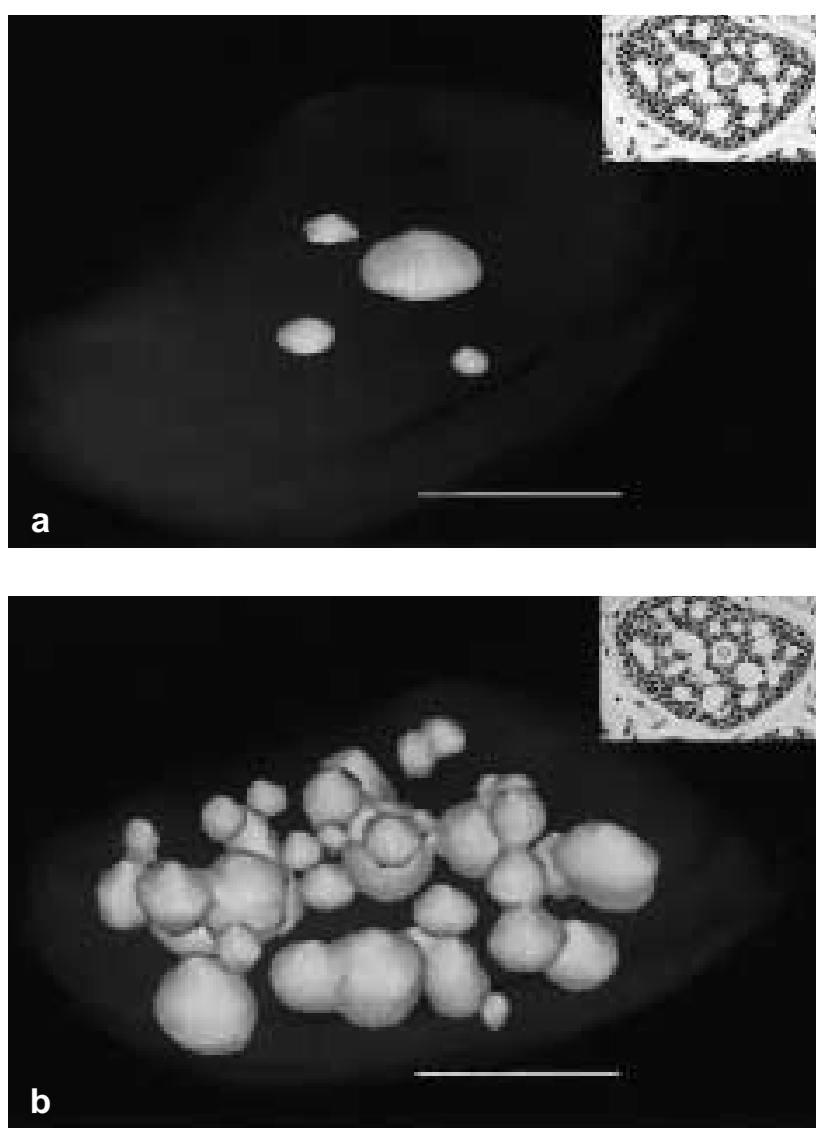

Fig. 3: (a) 3-D architecture of the true cystic spaces in the tumor cell nest of the cribriform pattern in ACC. bar $=100 \mu \mathrm{m}$ (Inset; 2-D figure, HE, $\times 35$ )

(b) 3-D architecture of the pseudocystic spaces in the same area. bar $=100 \mu \mathrm{m}$ (Inset; 2-D figure, HE, X35) 

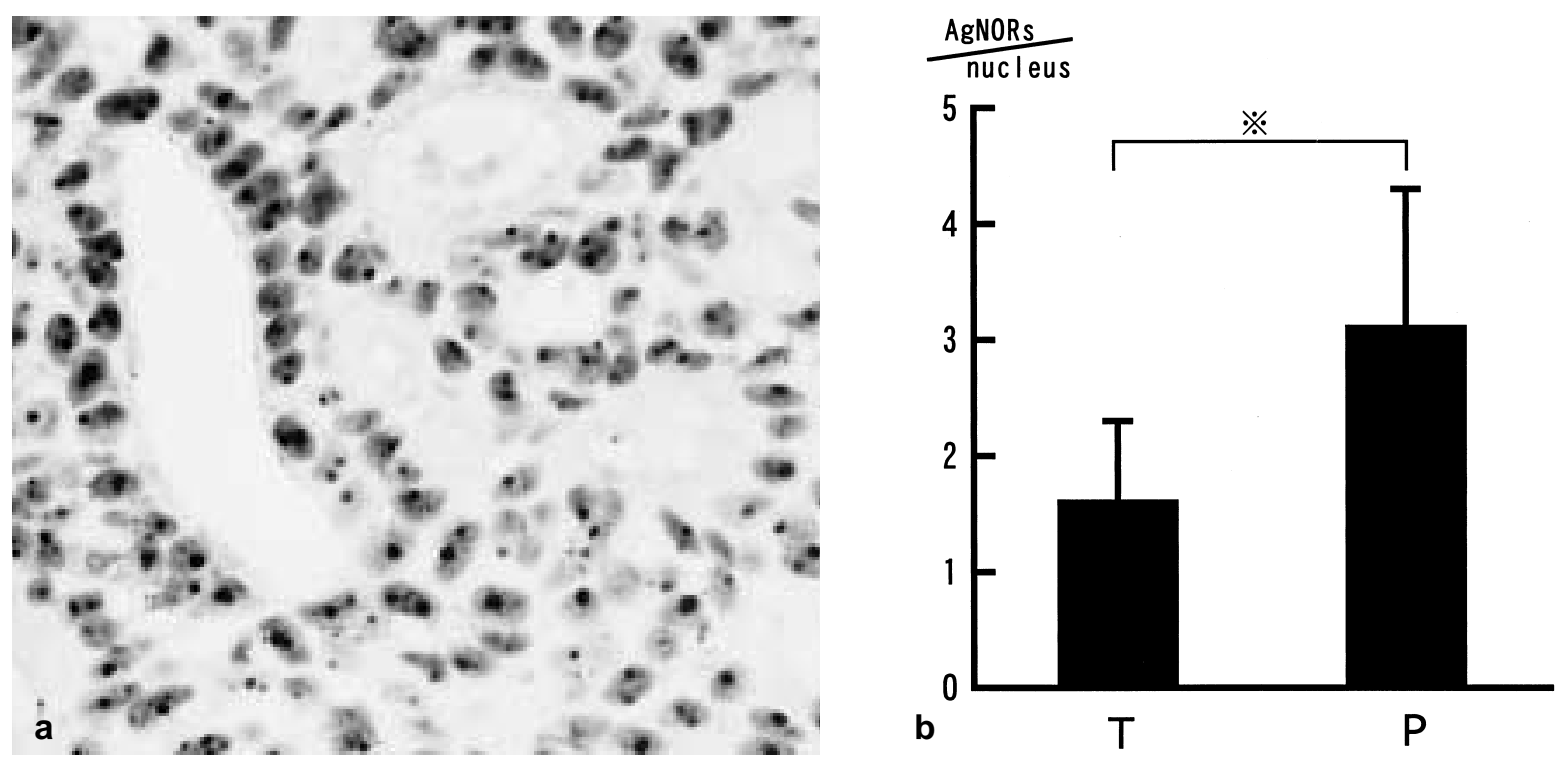

Fig. 4: (a) Tumor cell nuclei containing silver-stained AgNOR dots forming a true cyst (left) and several small pseudocysts (right). (AgNOR staining, $\times 540$ )

(b) The mean AgNOR number of tumor cells according to site. T: isolated true cyst, P: interconnecting pseudocyst. $(* ; p<0.001)$

\section{Discussion}

Although there have been many publications about cystic spaces in ACC by light $(10,11)$ and/or electron (10, 12-14) microscope, we have dealt with the characteristic from a morphological viewpoint. Bloom et al . (10) and Toida et al . (11) pointed out that the two types of cystic spaces, the true cyst and the pseudocyst, include contents with different histochemical properties. Electron microscopically, Tandler (12) revealed that the true cyst was surrounded by cuboidal cells showing microvilli of apical poles, whereas the pseudocyst was lined by highly replicated basal lamina. The present 3-D architecture of the cystic space suggests that the true cystic spaces may come to be larger with duct-like epithelial lining, whereas the pseudocystic spaces may become larger without a definitive epithelial lining and may be interconnected with each other and/or connected to the outer stroma. The different 3-D features between the true cyst and the pseudocyst may reflect the basic biological behavior of each cyst.

I mmunohistochemically, laminin has been shown to be confined to the basement membrane and can be used as a maker for the distinction between the true cyst and the pseudocyst forming the cribriform pattern in ACC (15-18). The true cyst is formed by ductal epitheliumlike cells that retains salivary secretory material within the lumen, whereas the pseudocyst is formed by myoepithelium-like cells and contains an extracellular matrix in its lumen $(19,20)$. The present study reveals that the proliferative status significantly increased in myoepithelium-like cells than ductal epithelium-like cells. These facts suggest that myoepithelium-like cells play an important role in the growth and biological behavior of ACC. Furthermore, Fujita et al . $(20,21)$ showed that apoptosis is easily induced in ductal epithelium-like cells rather than in myoepithelium-like cells. Therefore, myoepithelium-like cells forming the pseudocyst may be easier to reproduce than ductal epithelium-like cells forming the true cyst, and the tumor nest with predominant true cysts may transform into that with predominant pseudocysts. Chen et al . (7) proposed the following model of tumorigenesis of ACC: as neoplastic cells differentiate and proliferate, first the solid pattern is formed, next the cribriform or tubular pattern appears, and the latter two patterns may change into each other. Interestingly, they also indicated that the new tumor nest with a tubular pattern sprouts from the periphery of masses with a solid or cribriform pattern, and the outer cells of the tubular pattern, that is myoepithelium-like cells, may proliferate and form the pseudocysts. These findings may support the easy reproduction of myoepithelium-like cells forming the pseudocyst in comparison with ductal epithelium-like cells forming the true cyst.

The term "cribriform pattern" has been used in other diseases such as intraductal carcinoma of the breast (22), cribriform carcinoma of the prostate (23) and epididymal cribriform hyperplasia (24). Ohuchi et al . (22) pointed out the contrast between the cribriform pattern of carcinoma and the complex glandular pattern of the benign tumor of the breast by 3-D architecture. They reported that the cribriform pattern of the intraductal carcinoma seen in the 2-D figure corresponded to the porous structure observed in the 3-D architecture. The cribriform pattern of ACC in the present study was different from that of the intraductal carcinoma described by Ohuchi et al . (22) by 3-D architecture. It is therefore important to understand that there are great differences in the cribriform pattern of each neoplasm.

In conclusion, from the present 3-D feature of the 
cystic spaces in the tumor cell nests of ACC of salivary gland origin, the terms "sieve" and/or "cylinder" were not suitable for 3-D structural attributes. This study supports that these 3-D features were useful for a better understanding of biological characteristics of ACC.

\section{Acknowledgements}

We wish to thank Drs Yukiko Yamamura, Xu Hui and $\mathrm{Hu}$ J i-Min (Division of Oral Pathology, Department of Oral Medecine and Bioregulation, Tohoku University Graduate School of Dentistry) and Yoshinaka Shimizu (Division of Dental and Craniofacial Anatomy, Department of Oral Biology, Tohoku University Graduate School of Dentistry) for their helpful discussion. We also thank Mr Noriyoshi Sasaki for his skillful assistance.

\section{References}

1. Billroth T. Beobachtungen über Geschwulste der Speicheldrusen. Virchows Arch path Anat 1859; 17: 357-75.

2. Friedmann I and Osborn DA. The nasopharynx. In: Wright GP and Symmers WSC ed. Systemic Pathology, Vol.1. Longmans, London, 1966; 309-16.

3. Krompecher $\mathrm{E}$. Über die Beziehungen zwischen Epithel und Bindegewebe bei den Mischgeschwülsten der Haut und der Speicheldrüsen und über das Entstehen der Karcinosarkome. Beitr pathol Anat allgem Pathol 1908; 44: 88-149.

4. New GB and Childrey J H. Tumors of the tonsil and pharynx (three hundred and fifty-seven cases). II . Adenocarcinomas of the mixed tumor type (seventy-four cases). Arch Otolaryngol 1931; 14: 699-712.

5. Reid J D. Adenoid cystic carcinoma (cylindroma) of the bronchial tree. Cancer 1952; 5: 685-94.

6. Ploton $\mathrm{D}$, Menager $\mathrm{M}, \mathrm{J}$ eannesson $\mathrm{P}$, et al . Improvement in the staining and in the visualization of the argyrophilic proteins of the nucleolar organizer region at the optical level. HistochemJ 1986; 18: 5-14.

7. Chen J-C, Gnepp DR and Bedrossian CWM. Adenoid cystic carcinoma of the salivary glands: an immunohistochemical analysis. Oral Surg Oral Med Oral Pathol 1988; 65: 316-26.

8. Chomette G, Auriol M, Vaillant J M, et al . An immunohistochemical study of the distribution of lysozyme, lactoferrin, $\alpha$ 1-antitrypsin and $\alpha$ 1-antichymotrypsin in salivary adenoid cystic carcinoma. Pathol Res Pract 1991; 187: 1001-8.

9. Grenko RT, Abendroth CS, Davis AT, et al . Hybrid tumors or salivary gland tumors sharing common differentiation pathways? Reexamining adenoid cystic and epithelial-myoepithelial carcinomas. Oral Surg Oral Med Oral Pathol Oral Radiol Endod 1998; 86: 188-95.

10. Bloom GD, Carlsöö B, Gustafsson H, et al. Distribution of mucosubstances in adenoid cystic carcinoma. A light and electron microscopic study. Virchows Arch A Pathol Anat Histol 1977; 375: 1-12.

11. Toida M, Takeuchi J, Sobue M, et al . Histochemical studies on pseudocysts in adenoid cystic carcinoma of the human salivary gland. HistochemJ 1985; 17: 913-24.

12. Tandler B. Ultrastructure of adenoid cystic carcinoma of salivary gland origin. Lab Invest 1971; 24: 504-12.

13. Osborn DA. Morphology and the natural history of cribriform adenocarcinoma (adenoid cystic carcinoma). J Clin Pathol 1977; 30: 195-205.

14. Lawrence J B and Mazur MT. Adenoid cystic carcinoma: a comparative pathologic study of tumors in salivary gland, breast, lung, and cervix. Hum Pathol 1982; 13: 916-24.

15. Toida M, Takeuchi J , Hara K, et al . Histochemical studies of intercellular components of salivary gland tumors with special reference to glycosaminoglycan, laminin and vascular elements. Virchows Arch A Pathol Anat Histopathol 1984; 403: $15-26$.

16. d'Ardenne AJ , Kirkpatrick P, Wells CA, et al . Laminin and fibronectin in adenoid cystic carcinoma. J Clin Pathol 1986; 39: 138-44.

17. Yamada K, Kunikata M, Mori $M$, et al . Immunohistochemical localization of MAM-3 and MAM- 6 antigens in adenoid cystic carcinoma. J Oral Pathol Med 1991; 20: 57-63.

18. Cheng J , Saku T, Okabe H, et al. Basement membranes in adenoid cystic carcinoma. An immunohistochemical study. Cancer 1992; 69: 2631-40.

19. Dardick I and van Nostrand AW. Morphogenesis of salivary gland tumors. A prerequisite to improving classification. Pathol Annu 1987; 22: 1-53.

20. Fujita S, Shibata Y, Takahashi H, et al . Apoptosis-induced and -suppressed cells in salivary gland adenoid cystic carcinoma: correlation with histological growth patterns. Oral Dis 1999; 5: 117-22.

21. Fujita S, Takahashi $\mathrm{H}$ and Okabe H. Nucleolar organizer regions in malignant salivary gland tumors. Acta Pathol J pn 1992; 42: 727-33.

22. Ohuchi N, Abe R, Takahashi T, et al. Three-dimensional atypical structure in intraductal carcinoma differentiating from papilloma and papillomatosis of the breast. Breast Cancer Res Treat 1985; 5: 57-65.

23. Rubin MA, de La TailleA, Bagiella E, et al . Cribriform carcinoma of the prostate and cribriform prostatic intraepithelial neoplasia: incidence and clinical implications. Am J Surg Pathol 1998; 22: 840-8.

24. Sharp SC, Batt MA and Lennington WJ . Epididymal cribriform hyperplasia. A variant of normal epididymal histology. Arch Pathol Lab Med 1994; 118: 1020-2.

(Accepted for publication October 5, 2000) 